\title{
Dilations of Quantum Dynamical Semigroups with Classical Brownian Motion
}

\author{
R. Alicki ${ }^{\dagger}$ and M. Fannes ${ }^{\dagger \dagger}$ \\ Instituut voor Theoretische Fysica, Universiteit Leuven, B-3030 Leuven, Belgium
}

\begin{abstract}
We show that any quantum dynamical semigroup can be written with the help of the solution of a vector-valued classical stochastic differential equation. Moreover this equation leads to a natural construction of a unitary dilation in term of Wiener spaces.
\end{abstract}

\section{Introduction}

In this paper we study dilations of quantum dynamical semigroups. Such semigroups are the quantum analogues of classical Markov evolutions and they describe irreversibility without memory effects. The mathematical structure of quantum dynamical semigroups and their generators has been studied by many authors e.g. [1-4]. The dilation problem consists now in constructing a larger reversible dynamical system in such a way that the semigroup evolution arises as a reduced dynamics. The existence of dilations of quantum dynamical semigroups has been studied from a purely mathematical point of view in [5]. Recently, however, quite a few papers have been published in order to obtain explicit and physically relevant constructions of such dilations [6-8].

It is well known that classical Markov evolutions are generated by stochastic equations of Langevin type driven by Brownian motions. For quantum dynamical semigroups a construction of the following nature has been described by [9-11]. The quantum system is coupled to either Fermi or Bose fields of dimension 1 (both cases can be handled). Then a reversible dynamics of the composed system is constructed with the help of a quantum stochastic differential equation in the sense that not only the equation is operator-valued but also the noise terms are given by quantum fields. The fact that the noise terms do not commute is essential in this scheme in order to obtain a general quantum dynamical semigroup as reduced dynamics. Technically this approach uses therefore a non-commutative version of the Ito stochastic calculus.

Our approach is quite different [12]. We start from the observation that the 
quantum dynamical semigroup is generated by a stochastic differential equation on the vectors in the Hilbert space of the system; moreover it is sufficient to consider as driving term a multidimensional Brownian motion. The stochastic evolution of a vector in the system space can in general not be expected to be unitary. This is not surprising, also for the classical case the solution of the Langevin equation is not given in terms of canonical transformations of the phase-space of the system. However this stochastic evolution defines for a given initial state a vector-valued random variable $\phi_{t}(\omega), t \geqq 0$ with time independent $L^{2}$ norm. We show next that it is possible to construct a continuous unitary cocycle on the $L^{2}$ space of vector-valued random variables which maps the initial vector into its evolved random variable $\phi_{t}(\omega)$. Finally, composing this cocycle with the unitary shift we obtain a dilation of the quantum dynamical semigroup. Formally the generator of the unitary dilation group involves a very singular coupling between the system and the bath; for this reason we prefer to construct the evolution in terms of solutions of (ordinary) stochastic integral equations.

\section{Quantum Stochastic Equation with Classical Noise}

We denote by $\bar{B}=\left(B^{k}\right)_{k=1 \ldots d}$ the $d$-dimensional Brownian motion. Let $\Omega^{(d)}$ be the space of continuous sample paths on $\mathbb{R}$ with values in $\mathbb{R}^{d}$ and $\mathscr{B}$ the smallest Borel algebra generated by the sets $\left\{\omega \in \Omega^{(d)} \mid \omega(t)-\omega(s) \in A\right\}$, A Borel set of $\mathbb{R}^{d}, s<t \in \mathbb{R}$ and denote by $d P$ the Wiener probability measure defined by $\bar{B}$ on $\mathscr{B} . L^{2}\left(\Omega^{(d)}, d P\right)$ is then the Hilbert space of complex-valued $d P$ square integrable functions on $\Omega^{(d)}$ and $\left\{E_{s} \mid s \in \mathbb{R}\right\}$ is the family of conditional expectations which act on $L^{2}\left(\Omega^{(d)}, d P\right)$ as a strongly continuous family of orthogonal projections. We also write the scalar product in $L^{2}\left(\Omega^{(d)}, d P\right)$ as

$$
\langle f, g\rangle=\mathbb{E}(\bar{f} \cdot g), \quad f, g \in L^{2}\left(\Omega^{(d)}, d P\right),
$$

where $\mathbb{E}$ is the expectation with respect to the Brownian motion. The system will be described by a separable complex Hilbert space $\mathscr{H}$ and will be injected into $\mathscr{H} \otimes L^{2}\left(\Omega^{(d)}, d P\right)$ by

$$
\alpha \in \mathscr{H} \rightarrow \alpha \otimes e \in \mathscr{H} \otimes L^{2}\left(\Omega^{(d)}, d P\right),
$$

where $e$ is the unit function on $\Omega^{(d)}$. We will also use the notation $X \phi$ instead of $(X \otimes 1) \phi$ for $X \in \mathscr{B}(\mathscr{H}), \phi \in \mathscr{H} \otimes L^{2}\left(\Omega^{(d)}, d P\right)$.

We should also remark here that we use two different symbols for the scalar product in $\mathscr{H} \otimes L^{2}\left(\Omega^{(d)}, d P\right)$ :

$$
(\phi, \psi) \rightarrow\langle\phi \mid \psi\rangle \quad \text { and } \quad(\phi, \psi) \rightarrow \mathbb{E}(\langle\phi \mid \psi\rangle) .
$$

The first notation will be used for Hilbert space type arguments, whereas the second is used to make a connection with current practice in probability language. In the last case $\phi$ and $\psi$ are looked upon as $\mathscr{H}$-valued random variables and $\langle\phi \mid \psi\rangle$ denotes the scalar product in $\mathscr{H}$, hence $\langle\phi \mid \psi\rangle$ is now a complex-valued random variable on $\Omega^{(d)}$.

Next we consider a $d$-tuple $\bar{V}=\left\{V_{1}, \ldots, V_{d}\right\}$ of bounded linear operators on $\mathscr{H}$ 
and we use the following vector notation:

$$
\begin{aligned}
\bar{V}^{*} & =\left\{V_{1}^{*}, \ldots, V_{d}^{*}\right\}, \\
\bar{V} X \bar{V}^{*} & =\sum_{k=1}^{d} V_{k} X V_{k}^{*}, \quad X \in \mathscr{B}(\mathscr{H}), \\
\bar{V} d \bar{B}_{t} & =\sum_{k=1}^{d} V_{k} d B_{t}^{k}, \quad \text { etc. }
\end{aligned}
$$

Consider now the following stochastic differential equation on $\mathscr{H} \otimes L^{2}\left(\Omega^{(d)}, d P\right)$,

$$
d \phi_{t}=K \phi_{t} d t+\bar{V} \phi_{t} d \bar{B}_{t}, \quad t \geqq 0
$$

with initial condition $\phi_{0}=\alpha \otimes e, \alpha \in \mathscr{H}$, where $K \in \mathscr{B}(\mathscr{H})$. The solution $\phi_{t}, t \geqq 0$ is an adapted process which satisfies: $\forall \alpha \in \mathscr{H}$ and $t \geqq 0$,

$$
\mathbb{E}\left(\left\|\phi_{t}\right\|^{2}\right)=\|\alpha\|^{2}, \quad \text { iff } \quad K+K^{*}=-\bar{V}^{*} \bar{V} .
$$

The link between such stochastic differential equations and quantum dynamical semigroups is now given in

Proposition II.1. Let $\phi_{t}, t \geqq 0$ be the solution of Eq. (2) with initial condition $\alpha \otimes e$, $\alpha \in \mathscr{H}$, and suppose that $K \in \mathscr{B}(\mathscr{H})$ satisfies condition (3), then for all $X \in \mathscr{B}(\mathscr{H})$ :

$$
\mathbb{E}\left(\left\langle\phi_{t} \mid X \phi_{t}\right\rangle\right)=\left\langle\alpha \mid e^{t L}(X) \alpha\right\rangle
$$

where

$$
L(X)=K^{*} X+X K+\bar{V}^{*} X \bar{V}
$$

is a generator of a semigroup of completely positive unity preserving mappings on $\mathscr{B}(\mathscr{H})$ $[2,3]$.

Proof.

$$
\left|\mathbb{E}\left(\left\langle\phi_{t} \mid X \phi_{t}\right\rangle\right)\right| \leqq\|X\| \mathbb{E}\left(\left\|\phi_{t}\right\|^{2}\right)=\|X\| \quad\|\alpha\|^{2} .
$$

Hence there exists a unique function $t \in \mathbb{R}^{+} \rightarrow X_{t} \in \mathscr{B}(\mathscr{H})$ such that $\mathbb{E}\left(\left\langle\phi_{t} \mid X \phi_{t}\right\rangle\right)$ $=\left\langle\alpha \mid X_{t} \alpha\right\rangle$, where $X_{t} \in \mathscr{B}(\mathscr{H})$ and $X_{0}=X$. By Ito-calculus,

$$
\begin{aligned}
d\left\langle\alpha \mid X_{t} \alpha\right\rangle & =d \mathbb{E}\left(\left\langle\phi_{t} \mid X \phi_{t}\right\rangle\right)=\mathbb{E}\left(\left\langle d \phi_{t} \mid X \phi_{t}\right\rangle+\left\langle\phi_{t} \mid X d \phi_{t}\right\rangle+\left\langle d \phi_{t} \mid X d \phi_{t}\right\rangle\right) \\
& =\mathbb{E}\left(\left\langle K \phi_{t} d t \mid X \phi_{t}\right\rangle+\left\langle\phi_{t} \mid X K \phi_{t} d t\right\rangle+\left\langle\bar{V} \phi_{t} d \bar{B}_{t} \mid X \bar{V} \phi_{t} d \bar{B}_{t}\right\rangle\right) \\
& =\mathbb{E}\left(\left\langle\phi_{t} \mid\left(K^{*} X+X K+\bar{V}^{*} X \bar{V}\right) \phi_{t}\right\rangle\right) d t \\
& =\left\langle\alpha \mid(L X)_{t} \alpha\right\rangle d t,
\end{aligned}
$$

and therefore $X_{t}=e^{t L}(X), t \geqq 0$.

\section{Dilation of the Quantum Dynamical Semigroup}

In order to construct a unitary evolution on $\mathscr{H} \otimes L^{2}\left(\Omega^{(d)}, d P\right)$ which coincides for $t \geqq 0$ and the special initial condition $\phi_{0}=\alpha \otimes e, \alpha \in \mathscr{H}$ with the solution of Eq. (2), one has to introduce a modified Eq. (2). For a heuristic motivation of this new 
integral equation we refer the reader to [12]. We now first introduce new operators on $L^{2}\left(\Omega^{(d)}, d P\right)$.

For $t \geqq 0$ and $k=1, \ldots, d$, let

$$
\left(A_{t}^{k}\right)^{*} f=\int_{0}^{t} d B_{s}^{k} E_{s}(f), \quad f \in L^{2}\left(\Omega^{(d)}, d P\right)
$$

then

$$
\mathbb{E}\left(\left|\left(A_{t}^{k}\right)^{*} f\right|^{2}\right)=\mathbb{E}\left(\left|\int_{0}^{t} d B_{s}^{k} E_{s}(f)\right|^{2}\right)=\int_{0}^{t} d s \mathbb{E}\left(\left|E_{s}(f)\right|^{2}\right) \leqq t \mathbb{E}\left(|f|^{2}\right),
$$

and therefore $\left(A_{t}^{k}\right)^{*}, t \geqq 0$ is a bounded linear operator on $L^{2}\left(\Omega^{(d)}, d P\right)$ with norm

$$
\left\|\left(A_{t}^{k}\right)^{*}\right\|=\left\|\left(A_{t}^{k}\right)\right\|=t^{1 / 2} .
$$

We define also the free evolution $\left\{U_{t}^{0} \mid t \in \mathbb{R}\right\}$ on $L^{2}\left(\Omega^{(d)}, d P\right)$ by

$$
\left(\left(U_{t}^{0} f\right)(\omega)\right)(s)=(f(\omega))(s-t) \quad f \in L^{2}\left(\Omega^{(d)}, d P\right), \quad \omega \in \Omega^{(d)} \quad s, t \in \mathbb{R} .
$$

$\left\{U_{t}^{0} \mid t \in \mathbb{R}\right\}$ is a strongly continuous group of unitary operators on $L^{2}\left(\Omega^{(d)}, d P\right)$.

Lemma III.1. The following covariance relations hold:

$$
\begin{aligned}
U_{-t}^{0}\left(\bar{B}_{s_{1}}-\bar{B}_{s_{2}}\right) U_{t}^{0} & =\bar{B}_{s_{1}+t}-\bar{B}_{s_{2}+t}, & & s_{1}, s_{2}, t \in \mathbb{R}, \\
U_{-t}^{0} E_{s} U_{t}^{0} & =E_{s+t}, & & s, t \in \mathbb{R}, \\
U_{-t}^{0} \bar{A}_{s} U_{t}^{0} & =\bar{A}_{s+t}-\bar{A}_{t}, & & t \in \mathbb{R}^{+}, s \in \mathbb{R}^{+} .
\end{aligned}
$$

Proof. Follows immediately from the definitions.

We now introduce the evolution equation on $\mathscr{H} \otimes L^{2}\left(\Omega^{(d)}, d P\right)$. For $t \in[0, T]$, $T \geqq 0$, let $\mathscr{C}_{T}$ be the Banach space of continuous functions

$$
t \in[0, T] \rightarrow \phi_{t} \in \mathscr{H} \otimes L^{2}\left(\Omega^{(d)}, d P\right)
$$

equipped with the norm

$$
\|\mid \phi(\cdot)\|\left\|=\sup _{t \in[0, T]}\right\| \phi_{t} \| .
$$

We say that $\phi \in \mathscr{C}_{T}$ satisfies Eq. (12) with initial condition $\phi_{0} \in \mathscr{H} \otimes L^{2}\left(\Omega^{(d)}, d P\right)$, if for $t \in[0, T]$,

$$
\phi_{t}=\left(\mathbb{1}-\bar{A}_{t} \bar{V}^{*}\right) \phi_{0}+\int_{0}^{t} K E_{s}\left(\phi_{s}\right) d s+\int_{0}^{t} \bar{V} E_{s}\left(\phi_{s}\right) d \bar{B}_{s},
$$

where $K \in \mathscr{B}(\mathscr{H})$ satisfies condition(3).

\section{Theorem III.2.}

i) $\forall T>0$ and $\phi_{0} \in \mathscr{H} \otimes L^{2}\left(\Omega^{(d)}, d P\right) \exists$ ! solution $\phi_{t}$ of Eq. (12) with initial condition $\phi_{0}$,

ii) $\exists$ strongly continuous family $\left\{W_{t} \mid t \in \mathbb{R}\right\}$ of unitary operators on $\mathscr{H} \otimes L^{2}\left(\Omega^{(d)}, d P\right)$ such that $\phi_{t}=W_{t} \phi_{0}$, where $\phi_{t}$ satisfies (12) with initial condition $\phi_{0}$ for $t \geqq 0$,

iii) $W_{t_{1}+t_{2}}=U_{-t_{1}}^{0} W_{t_{2}} U_{t_{1}}^{0} W_{t_{1}} t_{1}, t_{2} \in \mathbb{R}$. Hence $\left\{U_{t} \equiv U_{t}^{0} W_{t} \mid t \in \mathbb{R}\right\}$ is a strongly continuous group of unitary operators on $\mathscr{H} \otimes L^{2}\left(\Omega^{(d)}, d P\right)$. 
Proof. The proof is divided in several steps. First of all we show that Eq. (12) admits locally (for small $T$ ) a unique solution (A); next we obtain the local cocycle relation in terms of the solution of (12) (B); then we obtain for small $T$ the existence of bounded linear operators $W_{t}$ such that $\phi(t)=W_{t} \phi_{0}$ and the local cocycle property for the $W_{t}$ holds (C); in (D) we show that those $W_{t}$ are isometric and in (E) that they are unitary and finally in $(\mathrm{F})$ we extend the $W_{t}$ to the whole of $\mathbb{R}$.

(A) We denote by $\|\bar{V}\|=\sum_{k=1}^{d}\left\|V_{k}\right\|$.

Let $T<T_{0}=\left(\left\{\|\bar{V}\|^{2}+4\|K\|\right\}^{1 / 2}-\|\bar{V}\|\right) / 2\|K\|$ and consider the iteration scheme

$$
\begin{aligned}
\phi_{t}^{(1)} & =\left(1-\bar{A}_{t} \bar{V}^{*}\right) \phi_{0} \quad \phi_{0} \in \mathscr{H} \otimes L^{2}\left(\Omega^{(d)}, d P\right) \\
\phi_{t}^{(n+1)} & =\left(1-\bar{A}_{t} \bar{V}^{*}\right) \phi_{0}+\int_{0}^{t} K E_{s}\left(\phi_{s}^{(n)}\right) d s+\int_{0}^{t} \bar{V} E_{s}\left(\phi_{s}^{(n)}\right) d \bar{B}_{s}, \quad n=1,2, \ldots
\end{aligned}
$$

From (7), (11) and the properties of the Ito integral it follows that $\phi_{(\cdot)}^{(n)} \in \mathscr{C}_{T}$, $n=1,2, \ldots$

Furthermore,

$$
\begin{aligned}
& \left\|\phi_{(\cdot)}^{(n+1)}-\phi_{(\cdot)}^{(n)}\right\| \| \\
& \quad=\sup _{t \in[0, T]}\left\|\int_{0}^{t} K E_{s}\left(\phi_{s}^{(n)}-\phi_{s}^{(n-1)}\right) d s+\int_{0}^{t} \bar{V} E_{s}\left(\phi_{s}^{(n)}-\phi_{s}^{(n-1)}\right) d \bar{B}_{s}\right\| \\
& \quad \leqq \sup _{t \in[0, T]}\left\{\left\|\int_{0}^{t} K E_{s}\left(\phi_{s}^{(n)}-\phi_{s}^{(n-1)}\right) d s\right\|+\left\|\int_{0}^{t} \bar{V} E_{s}\left(\phi_{s}^{(n)}-\phi_{s}^{(n-1)}\right) d \bar{B}_{s}\right\|\right\} \\
& \quad \leqq \sup _{t \in[0, T]}\|K\| \int_{0}^{t}\left\|\phi_{s}^{(n)}-\phi_{s}^{(n-1)}\right\| d s+\sup _{t \in[0, T]}\|\bar{V}\|\left\{\int_{0}^{t} d s\left\|\phi_{s}^{(n)}-\phi_{s}^{(n-1)}\right\|^{2}\right\}^{1 / 2} \\
& \quad \leqq\|K\| T\left\|\phi_{(\cdot)}^{(n)}-\phi_{(\cdot)}^{(n-1)}\right\|\|+\| \bar{V}\left\|T^{1 / 2}\right\| \phi_{(\cdot)}^{(n)}-\phi_{(\cdot)}^{(n-1)}\|\| \\
& \quad \leqq\left\{\|K\| T+\|\bar{V}\| T^{1 / 2}\right\}\left\|\phi_{(\cdot)}^{(n)}-\phi_{(\cdot)}^{(n-1)}\right\| \mid \leqq C\left\|\phi_{(\cdot)}^{(n)}-\phi_{(\cdot)}^{(n-1)}\right\| \|,
\end{aligned}
$$

where $C<1$ as $T<T_{0}$. Hence by the fixed point theorem (12) has a unique solution in $\mathscr{C}_{T}$.

(B)

Consider $t_{1}, t_{2} \in[0, T], \quad T<T_{0}, \quad$ such that $t_{1}+t_{2} \in[0, T]$ and for $\phi_{0} \in \mathscr{H} \otimes L^{2}\left(\Omega^{(d)}, d P\right)$ the equations

$$
\begin{aligned}
& \phi_{t}=\left\{1-\bar{A}_{t} \bar{V}^{*}\right\} \phi_{0}+\int_{0}^{t} K E_{s}\left(\phi_{s}\right) d s+\int_{0}^{t} \bar{V} E_{s}\left(\phi_{s}\right) d \bar{B}_{s}, \\
& \psi_{t}=\left\{1-\bar{A}_{t} \bar{V}^{*}\right\} U_{t_{1}}^{0} \phi_{t_{1}}+\int_{0}^{t} K E_{s}\left(\psi_{s}\right) d s+\int_{0}^{t} \bar{V} E_{s}\left(\psi_{s}\right) d \bar{B}_{s} .
\end{aligned}
$$

We next show that $\phi_{t_{1}+t_{2}}=U_{-t_{1}}^{0} \psi_{t_{2}}$.

In order to do so we need a few auxilliary results:

i) For $t, t_{1} \geqq 0$ and $f \in L^{2}\left(\Omega^{(d)}, d P\right)$,

$$
\left(A_{t+t_{1}}^{k}-A_{t_{1}}^{k}\right) A_{t_{1}}^{1} f=0, \quad k, 1 \in\{1, \ldots, d\} .
$$


Indeed for all $g \in L^{2}\left(\Omega^{(d)}, d P\right)$ one has

$$
\begin{aligned}
\left\langle g \mid\left(A_{t+t_{1}}^{k}-A_{t_{1}}^{k}\right) A_{t_{1}}^{1} f\right\rangle & =\left\langle\int_{t_{1}}^{t+t_{1}} d B_{s}^{k} E_{s}(g) \mid A_{t_{1}}^{1} f\right\rangle \\
& =\left\langle\int_{0}^{t_{1}} d B_{s^{\prime}}^{1} E_{s^{\prime}}\left(\int_{t_{1}}^{t+t_{1}} d B_{s}^{k} E_{s}(g)\right) \mid f\right\rangle=0 .
\end{aligned}
$$

ii) For $t, t_{1} \geqq 0$ and $f_{s}$ an adapted process on $L^{2}\left(\Omega^{(d)}, d P\right)$ one has

$$
\left(A_{t+t_{1}}^{k}-A_{t_{1}}^{k}\right) \int_{0}^{t_{1}} d s f_{s}=0, \quad k \in\{1, \ldots, d\} .
$$

Indeed for all $g \in L^{2}\left(\Omega^{(d)}, d P\right)$,

$$
\left\langle g \mid\left(A_{t+t_{1}}^{k}-A_{t_{1}}^{k}\right) \int_{0}^{t_{1}} d s f_{s}\right\rangle=\mathbb{E}\left\{\left(\int_{t_{1}}^{t+t_{1}} d B_{s^{\prime}}^{k} E_{s^{\prime}}(\bar{g})\right)\left(\int_{0}^{t_{1}} d s f_{s}\right)\right\}=0 .
$$

iii) For $t, t_{1} \geqq 0$ and $f_{s}$ and adapted process on $L^{2}\left(\Omega^{(d)}, d P\right)$

$$
\left(A_{t+t_{1}}^{k}-A_{t_{1}}^{k}\right) \int_{0}^{t_{1}} d B_{s}^{1} f_{s}=0, \quad k, 1 \in\{1, \ldots, d\} .
$$

Again, for $g \in L^{2}\left(\Omega^{(d)}, d P\right)$,

$$
\left\langle g \mid\left(A_{t+t_{1}}^{k}-A_{t_{1}}^{k}\right) \int_{0}^{t_{1}} d B_{s}^{1} f_{s}\right\rangle=\mathbb{E}\left\{\left(\int_{t_{1}}^{t+t_{1}} d B_{s^{\prime}} E_{s^{\prime}}(\bar{g})\left(\int_{0}^{t_{1}} d B_{s}^{1} f_{s}\right)\right\}=0 .\right.
$$

We now compute for $t>0$, using the covariance relations (9), (10) and (11) from Lemma III.1, (13), (14), and (15),

$$
\begin{aligned}
U_{-t_{1}}^{0} \psi(t)= & \phi_{t_{1}}-U_{-t_{1}}^{0} \bar{A}_{t} \bar{V}^{*} U_{t_{1}}^{0} \phi_{t_{1}}+U_{-t_{1}}^{0} \int_{0}^{t} K E_{s}\left(\psi_{s}\right) d s+U_{-t_{1}}^{0} \int_{0}^{t} \bar{V} E_{s}\left(\psi_{s}\right) d \bar{B}_{s} \\
= & \phi_{t_{1}}-\left(\bar{A}_{t+t_{1}}-\bar{A}_{t_{1}}\right) \bar{V}^{*} \phi_{t_{1}}+\int_{t_{1}}^{t+t_{1}} K E_{s}\left(U_{-t}^{0} \psi_{s-t_{1}}\right) d s \\
& +\int_{t_{1}}^{t+t_{1}} \bar{V} E_{s}\left(U_{-t_{1}}^{0} \psi_{s-t_{1}}\right) d \bar{B}_{s} \\
= & \left\{1-\left(\bar{A}_{t+t_{1}}-\bar{A}_{t_{1}}\right) \bar{V}^{*}\right\}\left\{\left(1-\bar{A}_{t_{1}} \bar{V}^{*}\right) \phi_{0}+\int_{0}^{t_{1}} K E_{s}\left(\phi_{s}\right) d s+\int_{0}^{t_{1}} \bar{V} E_{s}\left(\phi_{s}\right) d \bar{B}_{s}\right\} \\
& +\int_{t_{1}}^{t+t_{1}} K E_{s}\left(U_{-t_{1}}^{0} \psi_{s-t_{1}}\right) d s+\int_{t_{1}}^{t+t_{1}} \bar{V} E_{s}\left(U_{-t_{1}}^{0} \psi_{s-t_{1}}\right) d \bar{B}_{s} \\
= & \left\{1-\bar{A}_{t+t_{1}} \bar{V}^{*}\right\} \phi_{0}+\int_{0}^{t_{1}} K E_{s}\left(\phi_{s}\right) d s+\int_{t_{1}}^{t+t_{1}} K E_{s}\left(U_{-t_{1}}^{0} \psi_{s-t_{1}}\right) d s \\
& +\int_{0}^{t_{1}} \bar{V} E_{s}\left(\phi_{s}\right) d \bar{B}_{s}+\int_{t_{1}}^{t+t_{1}} \bar{V} E_{s}\left(U_{-t_{1}}^{0} \psi_{s-t_{1}}\right) d \bar{B}_{s} .
\end{aligned}
$$

Define now a new function $\tilde{\phi}(\cdot)$ in $\mathscr{C}_{T}$ :

$$
\begin{aligned}
\tilde{\phi}_{t} & =\phi_{t} \quad \text { for } 0 \leqq t \leqq t_{1} \\
& =U_{-t_{1}}^{0} \psi_{t-t_{1}} \quad \text { for } t>t_{1},
\end{aligned}
$$


then we just have shown that $\tilde{\phi}(\cdot)$ is a solution of (12); therefore by uniqueness we have $\tilde{\phi}_{t}=\phi_{t}, t \in[0, T]$, and so

$$
\phi_{t_{1}+t_{2}}=U_{-t_{1}}^{0} \psi_{t_{2}} .
$$

(C) From the iteration procedure in (A), we immediately obtain for $0 \leqq t \leqq T_{0}$ and $\phi_{0} \in \mathscr{H} \otimes L^{2}\left(\Omega^{(d)}, d P\right)$,

$$
\left\|\phi_{t}\right\| \leqq \frac{1+t^{1 / 2}\|\bar{V}\|}{1-\|K\| t-\|\bar{V}\| t^{1 / 2}}\left\|\phi_{0}\right\| .
$$

Therefore there exists for $0 \leqq t<T_{0}$ a family of bounded linear operators $W_{t}$ on $\mathscr{H} \otimes L^{2}\left(\Omega^{(d)}, d P\right)$ such that $W_{t} \phi_{0}=\phi_{t}$, where $\phi_{t}$ is the solution of (12) with initial condition $\phi_{0}$. As $\phi_{t} \in \mathscr{C}_{T}, W_{t}$ is strongly continuous in $t$ and satisfies by (B) the local cocycle relation

$$
W_{t_{1}+t_{2}}=U_{-t_{1}}^{0} W_{t_{2}} U_{t_{1}}^{0} W_{t_{1}}, \quad t_{1}, t_{2}, t_{1}+t_{2} \in[0, T] .
$$

(D) In order to show that $W_{t}, 0 \leqq t<T_{0}$, is an isometry we use the local cocycle relation (16):

$$
\begin{aligned}
\left\|W_{t+\varepsilon} \chi\right\|-\left\|W_{t} \chi\right\|= & \left\|U_{-t}^{0} W_{\varepsilon} U_{t}^{0} W_{t} \chi\right\|-\left\|U_{t}^{0} W_{t} \chi\right\| \\
= & \left\|W_{\varepsilon} U_{t}^{0} W_{t} \chi\right\|-\left\|U_{t}^{0} W_{t} \chi\right\|, \\
& \chi \in \mathscr{H} \otimes L^{2}\left(\Omega^{(d)}, d P\right) .
\end{aligned}
$$

It is therefore sufficient to show that for any $\phi_{0} \in \mathscr{H} \otimes L^{2}\left(\Omega^{(d)}, d P\right)$

$$
\lim _{\varepsilon \rightarrow 0} \frac{1}{\varepsilon}\left\{\left\|W_{\varepsilon} \phi_{0}\right\|-\left\|\phi_{0}\right\|\right\}=0 .
$$

We verify Eq. (17) by keeping in (12) all terms up to $o(\varepsilon)$,

$$
\begin{aligned}
W_{\varepsilon} \phi_{0}= & \phi_{0}-\bar{A}_{\varepsilon} \bar{V}^{*} \phi_{0}+\int_{0}^{\varepsilon} \bar{V} E_{s}\left(\phi_{0}\right) d \bar{B}_{s}+\int_{0}^{\varepsilon} K E_{s}\left(\phi_{0}\right) d s \\
& -\int_{0}^{\varepsilon} \bar{V} E_{s}\left(\bar{A}_{s} \bar{V} * \phi_{0}\right) d \bar{B}_{s}+\int_{0}^{\varepsilon} \bar{V} E_{s^{\prime}}\left(\int_{0}^{s^{\prime}} \bar{V} E_{s}\left(\phi_{0}\right) d \bar{B}_{s}\right) d \bar{B}_{s^{\prime}}+o(\varepsilon) .
\end{aligned}
$$

We then get:

$$
\begin{aligned}
\left\|W_{\varepsilon} \phi_{0}\right\|^{2}-\left\|\phi_{0}\right\|^{2} & =\left\|\bar{A}_{\varepsilon} \bar{V} \phi_{0}\right\|^{2}+\left\|\int_{0}^{\varepsilon} \bar{V} E_{s}\left(\phi_{0}\right) d \bar{B}_{s}\right\|^{2} \\
& +2 \operatorname{Re} \int_{0}^{\varepsilon} d s\left\langle\phi_{0} \mid K E_{s}\left(\phi_{0}\right)\right\rangle-2 \operatorname{Re}\left\langle\phi_{0} \mid \int_{0}^{\varepsilon} \bar{V} E_{s}\left(\overline{A_{s}} \bar{V} \phi_{0}\right) d \bar{B}_{s}\right\rangle .
\end{aligned}
$$

In order to avoid inessential notational complications we will continue the proof of (D) for a 1 dimensional Brownian motion. The general case can be treated in a similar way.

Any $\phi_{0} \in \mathscr{H} \otimes L^{2}\left(\Omega^{(1)}, d P\right)$ can be written uniquely as $\phi_{0}=\alpha \otimes e+\int \psi(s) d B_{s}$, where $\alpha \in \mathscr{H}$ and where $\psi(s)$ is a square integrable $\mathscr{H}$-valued adapted process. We 
now compute for $t \geqq 0$ the action of $A_{t}$ on $\phi_{0}$. For any $\chi \in \mathscr{H} \otimes L^{2}\left(\Omega^{(1)}, d P\right)$ :

$$
\begin{aligned}
\left\langle\chi \mid A_{t} \phi_{0}\right\rangle & =\left\langle A_{t}^{*} \chi \mid \phi_{0}\right\rangle=\left\langle\int_{0}^{t} E_{s}(\chi) d B_{s} \mid \phi_{0}\right\rangle=\left\langle\int_{0}^{t} E_{s}(\chi) d B_{s} \mid \alpha \otimes e \int \psi(s) d B_{s}\right\rangle \\
& =\int_{0}^{t}\left\langle E_{s}(\chi) \mid \psi(s)\right\rangle d s=\int_{0}^{t}\langle\chi \mid \psi(s)\rangle d s=\left\langle\chi \mid \int_{0}^{t} \psi(s) d s\right\rangle .
\end{aligned}
$$

Hence $A_{t} \phi_{0}=\int_{0}^{t} \psi(s) d s$.

We now use in (18) the following estimates:

$$
\begin{aligned}
\left\|A_{\varepsilon} V^{*} \phi_{0}\right\|^{2}= & \left\|\int_{0}^{\varepsilon} V^{*} \psi(s) d s\right\| \leqq \varepsilon \int_{0}^{\varepsilon}\left\|V^{*} \psi(s)\right\|^{2} d s \leqq \varepsilon\|V\|^{2} \int_{0}^{\varepsilon}\|\psi(s)\|^{2} d s=o(\varepsilon), \\
& \left|\left\langle\phi_{0} \mid \int_{0}^{\varepsilon} V E_{s}\left(A_{s} V^{*} \phi_{0}\right) d B_{s}\right\rangle\right| \leqq\left\|\phi_{0}\right\|\left\|\int_{0}^{\varepsilon} V E_{s}\left(A_{s} V^{*} \phi_{0}\right) d B_{s}\right\| \\
& =\left\|\phi_{0}\right\|\left\{\int_{0}^{\varepsilon} d s\left\|V A_{s} V^{*} \phi_{0}\right\|^{2}\right\}^{1 / 2}=o(\varepsilon) \quad \text { by (19). }
\end{aligned}
$$

Finally as $K+K^{*}=-V^{*} V$ it follows that $\left|\left\|W_{\varepsilon} \phi_{0}\right\|^{2}-\left\|\phi_{0}\right\|^{2}\right|=o(\varepsilon)$.

(E)

In order to prove that $W_{t}, 0 \leqq t<T_{0}$, is a unitary, it is by (D) sufficient to check that the range of $W_{t}$ is the whole of $\mathscr{H} \otimes L^{2}\left(\Omega^{(d)}, d P\right)$. From Eq. (12) and from the fact that $W_{t}, 0 \leqq t<T_{0}$, is an isometry we get

$$
\left\|\left(W_{t}-1\right) \phi_{0}\right\| \leqq\left\{\|K\| t+2\|\bar{V}\| t^{1 / 2}\right\}\left\|\phi_{0}\right\| .
$$

Hence $W_{t}$ is invertible on $\mathscr{H} \otimes L^{2}\left(\Omega^{(d)}, d P\right)$ for $t$ small enough. Using the local cocycle property (16) $W_{t}$ is unitary for $0 \leqq t<T_{0}$.

To end the proof one extends the local cocycle $W_{t}$ in the usual way. For $0<T<T_{0}$ and for $t \geqq 0 \exists N \in \mathbb{N}$ such that $N T \leqq t<(N+1) T$. Define then

$$
W_{t}=U_{-t}^{0}\left(W_{T} U_{T}^{0}\right)^{N} U_{t-N T}^{0} W_{t-N T}
$$

This definition is independent of $T$ due to the local cocycle property (16). For $t<0$ one puts $W_{t}=U_{-t}^{0}\left(W_{-t}\right)^{*} U_{t}^{0}$. It follows now immediately that $\left\{U_{t} \equiv U_{t}^{0} W_{t} \mid t \in \mathbb{R}\right\}$ has the properties stated in part iii) of the theorem.

Finally we remark that by the construction in (B) one has for all $t \geqq 0, W_{t} \phi_{0}=\phi_{t}$, where $\left\{\phi_{t} \mid t \geqq 0\right\}$ is the solution of (12) with initial condition $\phi_{0}$.

We are now coming to the main result of the paper. In the next theorem we show that the group $\left\{U_{t} \mid t \in \mathbb{R}\right\}$ constructed in Theorem III.2 dilates the quantum dynamical semigroup described in Proposition II.1.

\section{Theorem III.3.}

i) Let $\left\{\phi_{t} \mid t \geqq 0\right\}$ be the solution of the stochastic differential equation (2) with initial condition $\phi_{0}=\alpha \otimes e, \alpha \in \mathscr{H}$, then

$$
\phi_{t}=W_{t} \phi_{0} \quad t \geqq 0 .
$$


ii) For all $\alpha \in \mathscr{H}$ and $X \in \mathscr{B}(\mathscr{H})$,

$$
\left\langle\alpha \mid e^{t L}(X) \alpha\right\rangle=\left\langle\alpha \otimes e \mid U_{t}^{*} X U_{t} \alpha \otimes e\right\rangle,
$$

where $L$ is given as in (5).

\section{Proof.}

i) As $\left\{\phi_{t} \mid t \geqq 0\right\}$ is a solution of (2) with initial condition $\alpha \otimes e, \alpha \in \mathscr{H}, \phi_{t}$ is adapted. Plugging $\phi_{t}$ in Eq. (12), we get

$$
\left\{1-\bar{A}_{t} \bar{V}^{*}\right\} \phi_{0}+\int_{0}^{t} K E_{s}\left(\phi_{s}\right) d s+\int_{0}^{t} \bar{V} E_{s}\left(\phi_{s}\right) d \bar{B}_{s}=\phi_{0}+\int_{0}^{t} K \phi_{s} d s+\int_{0}^{t} \bar{V} \phi_{s} d \bar{B}_{s}=\phi_{t}
$$

by Eq. (2).

ii) By Proposition II.1,

$$
\begin{aligned}
\left\langle\alpha \mid e^{t L}(X) \alpha\right\rangle & =\mathbb{E}\left(\left\langle\phi_{t} \mid X \phi_{t}\right\rangle\right)=\left\langle W_{t} \phi_{0} \mid X W_{t} \phi_{0}\right\rangle \\
& =\left\langle U_{t}^{0} W_{t} \phi_{0} \mid X U_{t}^{0} W_{t} \phi_{0}\right\rangle=\left\langle\phi_{0} \mid U_{t}^{*} X U_{t} \phi_{0}\right\rangle .
\end{aligned}
$$

\section{References}

1. Davies, E. B.: Markovian master equations. Commun. Math. Phys. 39, 91 (1974)

2. Gorini, V., Kossakowski, A., Sudarshan, E. C. G.: Completely positive dynamical semigroups of $N$ level systems. J. Math. Phys. 17, 821 (1976)

3. Lindblad, G.: On the generators of quantum dynamical semigroups. Commun. Math. Phys. 48, 119 (1976)

4. Gorini, V., Frigerio A., Verri M., Kossakowski, A., Sudarshan, E. C. G.: Properties of quantum Markovian master equations. Rep. Math. Phys. 13, 149 (1978)

5. Evans, D. E., Lewis, J. T.: Dilations of irreversible evolutions in algebraic quantum theory. Commun. Dublin Inst. Adv. Study 24, Ser. A (1977)

6. Kümmerer, B.: A dilation theory for completely positive operators on $W^{*}$-algebras, Thesis, Tübingen (1982)

7. Accardi, L., Frigerio, A., Gorini, V., (eds.): Quantum probability and applications to the quantum theory of irreversible processes, Lecture Notes in Mathematics Vol. 1055, Berlin, Heidelberg, New York: Springer 1984

8. Accardi, L., von Waldenfels, W. (eds.): Quantum probability and applications II, Lecture Notes in Mathematics Vol. 1136, Berlin, Heidelberg, New York: Springer 1985

9. Barnett, C., Streater, R.F., Wilde, I.: J. Funct. Anal. 48, 172 (1982)

10. Hudson, R. L., Parthasarathy, K. R.: Time-orthogonal unitary dilations and non-commutative Feynman-Kac formulae. Commun. Math. Phys. 83, 301 (1984)

11. Applebaum, D. B., Hudson, R. L.: Fermion Ito's formula and stochastic evolutions. Commun. Math. Phys. 96, 473 (1984)

12. Alicki, R., Fannes, M.: On dilating quantum dynamical semigroups with classical brownian motion, Leuven preprint KUL-TF-85/17

Communicated by H. Araki

Received December 26, 1985; in revised form June 24, 1986 
